\title{
Rate of gestational weight gain and adverse pregnancy outcomes in rural nulliparous women: a prospective cohort analysis from China
}

\author{
Yubo Zhou ${ }^{1,2}$, Hongtian Li $^{1,2}$, Yali Zhang ${ }^{1,2}$, Le Zhang ${ }^{1,2}$, Jufen Liu ${ }^{1,2}$ and Jianmeng Liu ${ }^{1,2 *}$ \\ ${ }^{1}$ Institute of Reproductive and Child Health, Ministry of Health Key Laboratory of Reproductive Health, Peking University Health \\ Science Center, Beijing, People's Republic of China \\ ${ }^{2}$ Department of Epidemiology and Biostatistics, School of Public Health, Peking University Health Science Center, Beijing, \\ People's Republic of China \\ (Submitted 7 December 2018 - Final revision received 11 April 2019 - Accepted 7 May 2019; First published online 26 July 2019)
}

\section{Abstract}

Both inadequate and excessive gestational weight gain (GWG) have been shown to increase the risk of adverse pregnancy outcomes, but the risk profiles of GWG rate are unclear. We aimed to examine the associations between GWG rate in the second/third trimester and a spectrum of pregnancy outcomes. This study consisted of 14219 Chinese rural nulliparous women who participated in a randomised controlled trial of prenatal micronutrient supplementation during 2006-2009. The outcomes included stillbirth, neonatal and infant death, preterm birth, macrosomia, low birth weight (LBW) and large and small for gestational age (LGA and SGA, respectively). GWG rate was divided into quintiles within each BMI category. Compared with women in the middle quintile, those in the lowest quintile had higher risks of neonatal death (adjusted OR $2 \cdot 27 ; 95 \%$ CI 1.03, 5.02), infant death (adjusted OR 1.85; $95 \%$ CI 1.02, 3.37) and early preterm birth (adjusted OR 2.33; $95 \%$ CI 1.13, 4.77), while those in the highest quintile had higher risks of overall preterm birth (adjusted OR 1.28; $95 \%$ CI 1.04, 1.59), late preterm birth (adjusted OR 1.25; $95 \%$ CI 1.00, 1.56), LBW (adjusted OR 1.48; $95 \%$ CI 1.02, 2.15), macrosomia (adjusted OR 1.89; $95 \%$ CI 1.46, 2.45) and LGA (adjusted OR 1.56; 95 $\%$ CI $1 \cdot 31,1 \cdot 85)$. In conclusion, very low and very high GWG rates in the second/third trimester appear to be associated with adverse pregnancy outcomes in Chinese nulliparous women, indicating that an appropriate GWG rate during pregnancy is necessary for neonatal health.

Key words: Gestational weight gain: Stillbirth: Infant death: Preterm birth: Birth weight

Gestational weight gain (GWG), which is a modifiable factor influencing maternal and neonatal outcomes, remains a highly controversial topic, mainly reflected in the difficulty in balancing the risks of inadequate against excessive gain. Meta-analyses have shown that inadequate GWG is associated with increased risks of small for gestational age (SGA), low birth weight (LBW) and preterm birth $^{(1-3)}$, while excessive GWG is associated with increased risks of large for gestational age (LGA), macrosomia and caesarean delivery $^{(1,4)}$. However, previous studies had some limitations most studies used total GWG as the exposure measure, but few used rate of weight gain per week of gestation (GWG rate) ${ }^{(5-10)}$. Unlike total GWG, GWG rate does not heavily rely on the duration of pregnancy ${ }^{(11)}$, and thus is less likely to bias the association of GWG with pregnancy outcomes. An improved understanding of the association may provide insight into normative GWG in Chinese population. In addition, most studies focused on morbidity outcomes, and few dealt with mortality outcomes, such as neonatal and infant death ${ }^{(6,12-15)}$, which are of greater concern worldwide. Most previous studies were also conducted in
Caucasian populations, especially in American women, and there have been few such studies in non-Caucasian populations. The risk profiles between Caucasian and non-Caucasian populations might be different, as race-ethnicity factor was associated with both pattern of $\mathrm{GWG}^{(16)}$ and pregnancy outcomes ${ }^{(17)}$.

Therefore, we performed a prospective cohort analysis to comprehensively assess the associations of GWG rate in the second/third trimester with a spectrum of adverse pregnancy outcomes, including mortality and morbidity indicators, among Chinese nulliparous women.

\section{Methods}

\section{Study population}

Data for this prospective cohort analysis were retrieved from a randomised control trial (clinicaltrials.gov identifier: NCT00133744). The trial was conducted in five counties of Hebei province in China during 2006-2009 to investigate the

Abbreviations: GW, gestational week; GWG, gestational weight gain; IOM, Institute of Medicine; LBW, low birth weight; LGA, large for gestational age; SGA, small for gestational age.

* Corresponding author: Jianmeng Liu, fax +86-10-82805356, email liujm@pku.edu.cn 


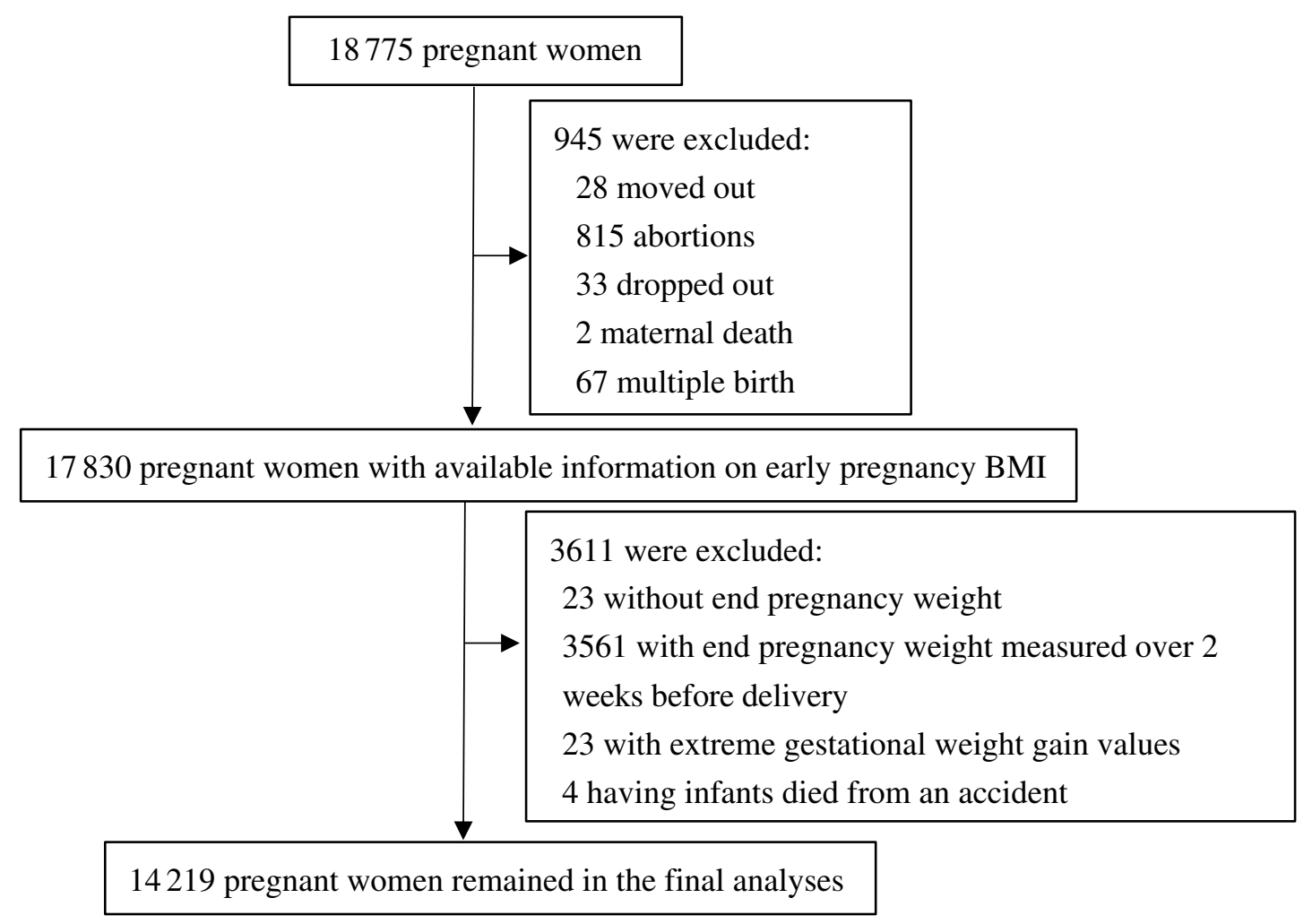

Fig. 1. Flowchart of study participants.

effects of prenatal micronutrient supplementation on pregnancy outcomes. The details of the trial, such as inclusion criteria of participants, have been presented elsewhere ${ }^{(18)}$. Briefly, 18775 nulliparous women were enrolled before gestational week (GW) 20 and individually randomised to receive a daily supplement containing folic acid, Fe-folic acid or multiple micronutrients from early pregnancy to delivery. The women were followed up monthly from enrolment through delivery, and their infants were followed up until 1 year after birth. A total of 4556 women were excluded due to moving out of the hospital catchment area ( $n$ 28), spontaneous or induced abortions ( $n$ 815), dropout ( $n$ 33), maternal death ( $n$ 2), multiple birth ( $n$ 67), missing end pregnancy weight ( $n$ 23), end pregnancy weight measured over 2 weeks prior to delivery ( $n 3561)$, extremely low ( $\leq 10 \mathrm{~kg}$ ) or extremely high ( $>35 \mathrm{~kg}$ ) GWG ( $n 23)$ or death of infant due to an accident $(n$ 4). Thus, 14219 women were included in the analysis (Fig. 1). There were no significant differences in demographic characteristics between the women who were excluded and those who were included in the study (Supplementary Table S1). Among the 14219 included women, 7378 were enrolled before GW 12, and 6841 were enrolled during GW 12-20. The demographic characteristics and adverse pregnancy outcomes were not substantially different between women enrolled before GW 12 and those enrolled during GW 12-20 (Supplementary Table S2). Women enrolled before GW 12 had slightly longer gestational age at delivery (39.6 v. $39 \cdot 4$ weeks), higher Hb concentration at enrolment $(126.4 v .122 .5 \mathrm{~g} / \mathrm{l})$ and lower rates of preterm ( $4.5 v .7 \cdot 6 \%)$ and LBW (1.9v. $2.4 \%)$ than those enrolled during GW 12-20. Because the GWG was lower before GW 12 but higher thereafter, different calculation methods for GWG rate in second/third trimester were applied in the following analyses.

The institutional review boards of the US Centers for Disease Control and Prevention (Atlanta, GA) and Peking University (Beijing, China) approved the trial. All women gave informed consent to participate in the trial. The analyses of already collected data were exempt by the institutional review boards.

\section{Rate of gestational weight gain}

The exposure measure used in the present analysis was rate of GWG in second/third trimester which is preferable over the total GWG, as the latter is intrinsically associated with gestational age $^{(11)}$ and, thus, with pregnancy outcomes, particularly preterm and birthweight outcomes. Because the GWG rate was low in first trimester, and high and linear in second/third trimester ${ }^{(16)}$, the GWG rate in second/third trimester was respectively calculated for women enrolled before GW 12 and those enrolled during GW 12-20. For women enrolled before GW 12, the GWG from enrolment to delivery was firstly calculated by subtracting the weight at enrolment from the end pregnancy weight, which was defined as the last recorded weight measured within 2 weeks before delivery. Then their exclusive GWG in second/ third trimester was determined as the difference between GWG from enrolment to delivery and the estimated first-trimester $\mathrm{GWG}^{(7)}$; the latter was calculated as: (12 weeks - GW at enrolment) $\times 0.11 \mathrm{~kg} /$ week, the GWG rate in first trimester for Asians $^{(16)}$. Finally, their GWG rate in second/third trimester was 
calculated by dividing the GWG in second/third trimester by the number of weeks between GW 12 and end-pregnancy weight. For women enrolled during GW 12-20, the GWG rate was calculated by subtracting the weight at enrolment from the end-pregnancy weight and then dividing by the number of weeks between the two measurements. As GWG rate differs with maternal BMI ${ }^{(19)}$, the GWG rate in second/third trimester was divided into quintiles within each BMI category.

Maternal weight and height were measured at enrolment before GW 20, using standardised equipment by trained staff. If weight at enrolment was used to calculate early-pregnancy BMI, some women enrolled during GW 12-20 would be classified into wrong BMI categories, due to the weight gain during GW 12-20. Therefore, for women enrolled during GW 12-20, we estimated the weight in first trimester as weight at enrolment minus estimated weight gain during GW 12-20; the latter was calculated as: (GW at enrolment -12 weeks) $\times 0.56 \mathrm{~kg} /$ week, the GWG rate in second trimester for Asians ${ }^{(16)}$. Early-pregnancy BMI $\left(\mathrm{kg} / \mathrm{m}^{2}\right)$ was estimated as first trimester weight in $\mathrm{kg}$ divided by the square of height in $\mathrm{m}$. Women were then classified into four BMI categories, according to the WHO guidelines for Asians ${ }^{(20)}$ : underweight, $<18.5 \mathrm{~kg} / \mathrm{m}^{2}$; normal weight, $18.5-23 \mathrm{~kg} / \mathrm{m}^{2}$; overweight, $23 \cdot 1-27.5 \mathrm{~kg} / \mathrm{m}^{2}$; and obese, $>27.5 \mathrm{~kg} / \mathrm{m}^{2}$.

Furthermore, an additional exposure measure was the rate of total GWG. The total GWG was firstly calculated by subtracting the first-trimester weight from the end-pregnancy weight, and the total GWG rate was calculated by dividing the total GWG by the number of weeks between the last menstrual period and the end-pregnancy weight ${ }^{(9)}$ and then divided into quintiles in the same manner as previously described. The mean total GWG of the included women was $12.9(4.7)$ and $11.5(4.6) \mathrm{kg}$ for their GWG in second/third trimester.

\section{Adverse pregnancy outcomes}

The outcomes of interest included mortality indicators and other adverse pregnancy outcomes. The mortality indicators included stillbirth, neonatal death, early neonatal death, perinatal death, infant death and total mortality from GW 28 to 1 year after birth. Stillbirth was defined as fetal death occurring on or after GW 28. Neonatal death was defined as death occurring within $28 \mathrm{~d}$ after birth, early neonatal death as death occurring within $7 \mathrm{~d}$ after birth, and infant death as death occurring within $365 \mathrm{~d}$ after birth. Perinatal death was defined as the sum of stillbirth plus early neonatal death, and total mortality as the sum of stillbirth plus infant death. All mortality events were determined through active community surveillance and validated by registration certificates or hospital records ${ }^{(18)}$.

The other adverse pregnancy outcomes included preterm birth, LBW, macrosomia, SGA and LGA. Preterm birth was defined as live birth within 37 completed GW from the first day of the last menstrual period; early preterm birth as birth occurring within 32 completed GW; and late preterm birth as birth occurring within 32-37 completed GW. LBW was defined as birth weight $<2500 \mathrm{~g}$, and macrosomia as birth weight $\geq 4000 \mathrm{~g}$. SGA and LGA were defined as birth weight below the 10th percentile for gestational age and above the 90th percentile for gestational age, respectively.

\section{Statistical analysis}

Continuous variables are presented as means and standard deviations, while categorical variables are shown as numbers and percentages. Differences across the GWG rate quintiles were assessed by ANOVA for continuous variables and the $\chi^{2}$ test for categorical variables. Multivariable logistic regression models were used to estimate the adjusted OR and $95 \%$ CI for pregnancy outcomes across different GWG rate quintiles, with the middle quintile serving as the reference group. In multivariable models, we adjusted for maternal age $(<25,25-29$ or $\geq 30$ years), educational level (high school or above, middle school, or elementary school or less), occupation (farmer or other), ethnicity (Han or other), micronutrient supplementation (folic acid, Fe-folic acid or multiple micronutrients), BMI in early pregnancy $\left(<18.5, \quad 18.5-23,23.1-27.5\right.$ or $\left.>27.5 \mathrm{~kg} / \mathrm{m}^{2}\right)$, Hb level at enrolment (100-109, 110-119, 120-129 or $\geq 130$ $\mathrm{g} / \mathrm{l}$ ) and gestational age at enrolment (as a continuous variable). We further adjusted for gestational age at delivery $(<37$ or $\geq 37$ weeks) in models for mortality indicators, LBW and macrosomia. When we estimated the adjusted OR and 95\% CI for a given pregnancy outcome, only women at risk of that outcome were taken into consideration. That is, all 14219 women were included in analyses of stillbirth, then women whose pregnancy ended in stillbirth ( $n$ 49) were excluded from analyses of other pregnancy outcomes.

To assess the robustness of our results, we repeated the main analyses using the rate of total GWG. To compare estimates of association with those reported in previous studies, we further repeated our main analyses using the recommended GWG rates in the second/third trimester provided in the 2009 Institute of Medicine (IOM) guidelines ${ }^{(19)}$. Women were then divided into three groups: less than, within or greater than the guideline in each BMI category, stratified as follows: underweight, $<18.5$ $\mathrm{kg} / \mathrm{m}^{2}$; normal weight, 18.5 to $<25 \mathrm{~kg} / \mathrm{m}^{2}$; overweight, 25 to $<$ $30 \mathrm{~kg} / \mathrm{m}^{2}$; and obese, $\geq 30 \mathrm{~kg} / \mathrm{m}^{2}$. All analyses were performed with SPSS software (version 20.0; SPSS Inc.). Statistical tests were two-sided, and $P<0.05$ was taken to indicate statistical significance.

\section{Results}

The mean maternal BMI in early pregnancy was 21.9 (sD 2.8) kg/ $\mathrm{m}^{2}$. Among the 14219 nulliparous women, 1187 (8.3\%) were categorised as underweight, 8897 (62.6 \%) as normal weight, $3522(24.8 \%)$ as overweight and $613(4.3 \%)$ as obese. Overall, the mean rate of GWG in the second/third trimester was $0.47(\mathrm{sD} \mathrm{0.19)} \mathrm{kg} /$ week, with values of $0.58(\mathrm{sD} 0.23) \mathrm{kg} /$ week for underweight, 0.48 (SD $0 \cdot 18$ ) $\mathrm{kg} /$ week for normal weight, $0.42(\mathrm{SD} 0.18) \mathrm{kg} /$ week for overweight and 0.38 (SD 0.19) $\mathrm{kg} /$ week for obese (Table 1). Maternal characteristics, including maternal age, height, education level, occupation, gestational age at enrolment and $\mathrm{Hb}$ level at enrolment across quintile groups of GWG rate in second/third trimester, are shown in Table 2.

Among all women included in this study, forty-nine (3.4\%o) pregnancies ended in stillbirth, while 14170 ended in live births. Among the live births, there were sixty-two ( $4.4 \%$ ) early 
Table 1. Characteristics of gestational weight gain (GWG) rate and Institute of Medicine (IOM) guidelines in second/third trimester (Mean values and standard deviations)

\begin{tabular}{|c|c|c|c|c|c|c|c|c|c|c|c|c|c|c|c|c|}
\hline \multirow[b]{3}{*}{ Early-pregnancy BMI* } & \multicolumn{5}{|c|}{ Overall } & \multicolumn{10}{|c|}{ GWG rate in second/third trimester by quintiles (kg/week) } & \multirow[b]{3}{*}{ IOM guidelines } \\
\hline & \multirow[b]{2}{*}{$n$} & \multicolumn{2}{|c|}{$\begin{array}{l}\text { GWG in } \\
\text { second/third } \\
\text { trimester } \\
(\mathrm{kg})\end{array}$} & \multicolumn{2}{|c|}{$\begin{array}{l}\text { GWG rate } \\
\text { in second/ } \\
\text { third } \\
\text { trimester } \\
\text { (kg/week) }\end{array}$} & \multicolumn{2}{|c|}{ Lowest } & \multicolumn{2}{|c|}{ Lower } & \multicolumn{2}{|c|}{ Middle } & \multicolumn{2}{|c|}{ Higher } & \multicolumn{2}{|c|}{ Highest } & \\
\hline & & Mean & SD & Mean & SD & Mean & SD & Mean & SD & Mean & SD & Mean & SD & Mean & SD & \\
\hline Underweight & 1187 & $13 \cdot 78$ & 5.32 & 0.58 & 0.23 & 0.31 & 0.07 & 0.45 & 0.03 & 0.55 & 0.03 & 0.66 & 0.04 & 0.93 & 0.19 & $0.44-0.58$ \\
\hline Normal weight & 8897 & 11.79 & 4.45 & 0.48 & 0.18 & 0.26 & 0.08 & 0.38 & 0.02 & 0.46 & 0.02 & 0.55 & 0.03 & 0.75 & 0.14 & $0.35-0.50$ \\
\hline Overweight & 3522 & $10 \cdot 45$ & 4.39 & 0.42 & 0.18 & 0.19 & 0.11 & 0.34 & 0.02 & 0.41 & 0.02 & 0.49 & 0.03 & 0.67 & 0.13 & $0.23-0.33$ \\
\hline Obese & 613 & $9 \cdot 39$ & 4.71 & 0.38 & 0.19 & 0.13 & 0.14 & 0.30 & 0.03 & 0.38 & 0.02 & 0.46 & 0.03 & 0.63 & 0.11 & $0.17-0.27$ \\
\hline Total & 14219 & 11.52 & 4.63 & 0.47 & 0.19 & 0.24 & $0 \cdot 10$ & 0.37 & 0.04 & 0.45 & 0.04 & 0.54 & 0.06 & 0.74 & $0 \cdot 16$ & NA \\
\hline
\end{tabular}

Table 2. Maternal characteristics according to quintiles of gestational weight gain (GWG) rate in second/third trimester (Numbers and percentages; mean values and standard deviations)

\begin{tabular}{|c|c|c|c|c|c|c|c|c|c|c|c|}
\hline & \multicolumn{10}{|c|}{ Quintiles of GWG rate in second/third trimester ( $n 14$ 219) } & \multirow[b]{3}{*}{$P$} \\
\hline & \multicolumn{2}{|c|}{$\begin{array}{l}\text { Lowest } \\
\text { (n2855) } \\
\end{array}$} & \multicolumn{2}{|c|}{$\begin{array}{c}\text { Lower } \\
(n \text { 2812) } \\
\end{array}$} & \multicolumn{2}{|c|}{$\begin{array}{c}\text { Middle } \\
\text { (n 2861) }\end{array}$} & \multicolumn{2}{|c|}{$\begin{array}{c}\text { Higher } \\
(n \text { 2851) }\end{array}$} & \multicolumn{2}{|c|}{$\begin{array}{l}\text { Highest } \\
(n \text { 2840) }\end{array}$} & \\
\hline & $n$ & $\%$ & $n$ & $\%$ & $n$ & $\%$ & $n$ & $\%$ & $n$ & $\%$ & \\
\hline Maternal age (years) & & & & & & & & & & & 0.90 \\
\hline$<25$ & 2068 & 72.4 & 2020 & 71.8 & 2060 & $72 \cdot 0$ & 2067 & $72 \cdot 5$ & 2079 & $73 \cdot 2$ & \\
\hline 25 to $<30$ & 680 & 23.8 & 684 & $24 \cdot 3$ & 687 & $24 \cdot 0$ & 663 & $23 \cdot 3$ & 645 & $22 \cdot 7$ & \\
\hline$\geq 30$ & 107 & 3.8 & 108 & 3.8 & 114 & 4.0 & 121 & $4 \cdot 2$ & 116 & $4 \cdot 1$ & \\
\hline Maternal height $(\mathrm{cm})$ & & & & & & & & & & & $0.00^{\star \star \star}$ \\
\hline$\leq 155$ & 352 & $12 \cdot 3$ & 346 & $12 \cdot 3$ & 418 & $14 \cdot 6$ & 490 & $17 \cdot 2$ & 573 & $20 \cdot 2$ & \\
\hline$>155-160$ & 1163 & $40 \cdot 7$ & 1267 & $45 \cdot 1$ & 1307 & $45 \cdot 7$ & 1366 & 47.9 & 1267 & 44.6 & \\
\hline$>160-165$ & 1010 & $35 \cdot 4$ & 899 & $32 \cdot 0$ & 879 & 30.7 & 777 & $27 \cdot 3$ & 765 & $26 \cdot 9$ & \\
\hline$>165$ & 330 & $11 \cdot 6$ & 300 & $10 \cdot 7$ & 257 & $9 \cdot 0$ & 218 & $7 \cdot 6$ & 235 & $8 \cdot 3$ & \\
\hline Education & & & & & & & & & & & $0.00^{* *}$ \\
\hline High school or above & 525 & $18 \cdot 4$ & 507 & $18 \cdot 0$ & 511 & $17 \cdot 9$ & 422 & $14 \cdot 8$ & 541 & $19 \cdot 0$ & \\
\hline Middle school & 2291 & $80 \cdot 2$ & 2274 & $80 \cdot 9$ & 2317 & $81 \cdot 0$ & 2379 & 83.4 & 2237 & $78 \cdot 8$ & \\
\hline Primary school or less & 39 & 1.4 & 31 & $1 \cdot 1$ & 33 & 1.1 & 50 & 1.8 & 62 & $2 \cdot 2$ & \\
\hline Occupation & & & & & & & & & & & $0.00^{* *}$ \\
\hline Farmer & 2606 & 91.3 & 2568 & 91.3 & 2671 & 93.4 & 2638 & 92.5 & 2548 & 89.7 & \\
\hline Other & 249 & 8.7 & 244 & $8 \cdot 7$ & 190 & $6 \cdot 6$ & 213 & 7.5 & 292 & $10 \cdot 3$ & \\
\hline Ethnicity & & & & & & & & & & & 0.59 \\
\hline Han & 2826 & 99.0 & 2784 & 99.0 & 2822 & 98.6 & 2815 & 98.7 & 2803 & $98 \cdot 7$ & \\
\hline Other & 29 & 1.0 & 28 & 1.0 & 39 & 1.4 & 36 & 1.3 & 37 & 1.3 & \\
\hline \multicolumn{12}{|c|}{ Gestational age at enrolment (weeks) $\dagger$} \\
\hline Mean & \multirow{2}{*}{\multicolumn{2}{|c|}{$\begin{array}{c}11.3 \\
4.4\end{array}$}} & \multirow{2}{*}{\multicolumn{2}{|c|}{$\begin{array}{c}11.6 \\
4.4\end{array}$}} & \multirow{2}{*}{\multicolumn{2}{|c|}{$\begin{array}{c}12.2 \\
4.5\end{array}$}} & \multirow{2}{*}{\multicolumn{2}{|c|}{$\begin{array}{c}12 \cdot 3 \\
4.5\end{array}$}} & \multirow{2}{*}{\multicolumn{2}{|c|}{$\begin{array}{c}13.4 \\
4.7\end{array}$}} & $0.00^{\star \star \star}$ \\
\hline SD & & & & & & & & & & & \\
\hline$<12$ & 1743 & $61 \cdot 0$ & 1580 & $56 \cdot 2$ & 1456 & $50 \cdot 9$ & 1458 & $51 \cdot 1$ & 1141 & $40 \cdot 2$ & $0.00^{\star * *}$ \\
\hline $12-20$ & 1112 & 39.0 & 1232 & $43 \cdot 8$ & 1405 & $49 \cdot 1$ & 1393 & 48.9 & 1699 & 59.8 & \\
\hline \multicolumn{12}{|c|}{ Gestational age at delivery (weeks) } \\
\hline$<37$ & 179 & $6 \cdot 3$ & 165 & $5 \cdot 8$ & 165 & 5.8 & 150 & $5 \cdot 3$ & 213 & 7.5 & $0.00^{\star \star}$ \\
\hline$\geq 37$ & 2676 & 93.7 & 2647 & $94 \cdot 1$ & 2696 & $94 \cdot 2$ & 2701 & 94.7 & 2627 & 92.5 & \\
\hline $\mathrm{Hb}$ at enrolment $(\mathrm{g} / \mathrm{l})$ & & & & & & & & & & & $0.00^{\star * \star}$ \\
\hline $100-109$ & 144 & $5 \cdot 0$ & 118 & $4 \cdot 2$ & 152 & $5 \cdot 3$ & 135 & 4.7 & 200 & $7 \cdot 0$ & \\
\hline $110-119$ & 598 & $21 \cdot 0$ & 608 & $21 \cdot 6$ & 623 & $21 \cdot 8$ & 678 & $23 \cdot 8$ & 730 & $25 \cdot 7$ & \\
\hline $120-129$ & 1267 & 44.4 & 1250 & 44.5 & 1238 & 43.3 & 1221 & $42 \cdot 8$ & 1139 & 40.1 & \\
\hline$\geq 130$ & 846 & $29 \cdot 6$ & 836 & $29 \cdot 7$ & 848 & $29 \cdot 6$ & 817 & 28.7 & 771 & $27 \cdot 2$ & \\
\hline Micronutrient supplementation & & & & & & & & & & & 0.89 \\
\hline Folic acid & 936 & $32 \cdot 8$ & 943 & 33.5 & 943 & 33.0 & 946 & 33.2 & 967 & 34.0 & \\
\hline Fe-folic acid & 969 & 33.9 & 949 & $33 \cdot 8$ & 973 & $34 \cdot 0$ & 961 & 33.7 & 911 & $32 \cdot 1$ & \\
\hline Multiple micronutrients & 950 & 33.3 & 920 & $32 \cdot 7$ & 945 & 33.0 & 944 & 33.1 & 962 & 33.9 & \\
\hline
\end{tabular}

${ }^{* *} P<0.01,{ }^{* \star *} P<0.001$

† Because gestational age at enrolment was adjusted as a continuous variable, both its means and standard deviations, and $n$ and $\%$ are presented. 
neonatal deaths, seventy-six (5.4\%) neonatal deaths and 109 (7.7 \%o) infant deaths. Of the seventy-six neonatal deaths, twenty-two died of asphyxia, twenty-two of premature birth, fifteen had birth defects, ten had infection, and seven died of other or unknown causes; the corresponding numbers for the 109 infant deaths were twenty-seven, twenty-two, twenty-seven, seventeen and sixteen, respectively. The associations between GWG rate in the second/third trimester and mortality indicators are presented in Table 3. Compared with women in the middle quintile, those in the lowest quintile had higher risks of infant death (adjusted OR 1.85; $95 \%$ CI 1.02, 3.37) and neonatal death (adjusted OR 2.27; $95 \%$ CI 1.03, 5.02), and those in the lower quintile also had a higher risk of neonatal death (adjusted OR 2.34; $95 \%$ CI 1.06, 5.17).

Of the live births, $845(6.0 \%)$ were preterm, $307(2.2 \%)$ were LBW, 570 ( $4.0 \%)$ had macrosomia, 1366 (9.6 \%) were SGA and 1383 ( $9.8 \%)$ were LGA. The associations between GWG rate in second/third trimester and these adverse outcomes are shown in Table 4. Compared with women in the middle quintile, those in the lowest quintile had increased risks of early preterm birth (adjusted OR 2.33; $95 \%$ CI 1.13, 4.77), while those in the highest quintile had increased risks of overall preterm birth (adjusted OR 1.28; $95 \%$ CI 1.04, 1.59), late preterm birth (adjusted OR 1.25; $95 \%$ CI 1.00, 1.56), macrosomia (adjusted OR 1.89; $95 \%$ CI 1.46, 2.45), LGA (adjusted OR 1.56; $95 \%$ CI 1.31, 1.85) and LBW (adjusted OR 1.48; $95 \%$ CI $1 \cdot 02,2 \cdot 15$ ).

When we repeated the main analysis using the total GWG rate, the results regarding preterm and birthweight outcomes were not substantially changed, while no positive association was found for mortality indicators (Supplementary Table S3).

In an analysis of GWG rate in the second/third trimester classified according to the 2009 IOM guidelines, the proportions of women with weight gain less than, within and greater than the recommendations were $23 \cdot 0,35 \cdot 7$ and $41.3 \%$, respectively. In comparison with women who showed weight gain within the recommendation, those with less weight gain had increased risks of offspring total mortality (adjusted OR 1.55; $95 \%$ CI 1.04, 2.32), infant death (adjusted OR 1.63; $95 \%$ CI 1.01, 2.63), LBW (adjusted OR 1.41; $95 \%$ CI 1.02, 1.94) and SGA (adjusted OR 1.15; $95 \%$ CI 1.01, 1.31), while those with greater weight gain showed increased risks of macrosomia (adjusted OR $1 \cdot 38 ; 95 \%$ CI $1 \cdot 13,1 \cdot 67$ ) and LGA (adjusted OR $1 \cdot 28 ; 95 \%$ CI $1 \cdot 12$, 1.45) (Supplementary Table S4).

\section{Discussion}

In this prospective cohort analysis of nulliparous Chinese women, the GWG rate in the second/third trimester was associated with increased risks of various adverse pregnancy outcomes, independent of maternal BMI in early pregnancy. In comparison with women in the middle quintile of GWG rate in second/third trimester, those in the lowest quintile had increased risks of infant death, neonatal death and early preterm birth, while those in the highest quintile had increased risks of preterm birth, macrosomia, LGA and LBW. 
Previous studies regarding infant mortality were limited by the use of either total GWG ${ }^{(12-15)}$ or GWG rate ${ }^{(6)}$ as the exposure measure. All of these studies were performed in American women, but the results were inconsistent. One case-control study $^{(6)}$ and one retrospective cohort study ${ }^{(12)}$ using selfreported GWG indicated that, in comparison with average GWG rate or GWG z-scores, low and high GWG were associated with an increased risk of infant death, mainly driven by early neonatal $^{(12)}$ or neonatal death ${ }^{(6)}$. The third study reported an increased risk of infant death in association with inadequate GWG, but a lower risk with excessive GWG, in comparison with GWG within the 2009 IOM guidelines ${ }^{(13)}$. The two remaining studies did not observe any positive associations for neonatal or infant death ${ }^{(14,15)}$. In our study of nulliparous Chinese women, we also found increased risks of infant death and neonatal death for the lowest quintile, but not for the highest quintile, compared with the middle quintile of GWG rate in second/third trimester. Although the association of GWG rate with causespecific death were not studied due to the relatively small number of cases, the primary causes of neonatal or infant death were asphyxia, premature birth, birth defects (such as congenital heart defects) and infection.

Our findings regarding birth weight outcomes were partially consistent with previous studies. A recent meta-analysis using total GWG showed a higher risk of SGA in association with inadequate GWG, and higher risks of macrosomia and LGA in association with excessive GWG, in comparison with GWG within the IOM guidelines ${ }^{(1)}$. Studies using the second/ third-trimester GWG rate also reported an increased risk of SGA with inadequate GWG rate, and of LGA with excessive GWG rate, among American and Brazilian women ${ }^{(7,21)}$. Inadequate or excessive GWG rate may reflect exposure of the foetus to reduced or greater amounts of glucose and fatty acids during development, respectively ${ }^{(22,23)}$. In our study, we found higher risks of LGA and macrosomia for the highest quintile, but not of SGA for the lowest quintile among nulliparous Chinese women. Unexpectedly, we found an increased risk of LBW for the highest quintile of GWG rate. We wonder whether it reflects a true association, as it might be biased by gestational age at enrolment. For example, women enrolled at larger gestational age were more likely to have a higher GWG rate in second/third trimester and a higher rate of LBW. Although we had adjusted gestational age at enrolment as a continuous variable, a residual confounding might have still remained. Additionally, we did not find any positive association between GWG rate and LBW when the total GWG or the second/third-trimester GWG rates classified according to the 2009 IOM guidelines were used.

Results regarding the association between GWG and preterm birth reported to date are not consistent across studies. One meta-analysis indicated that total GWG below the IOM guideline was associated with a higher risk of preterm birth, while total GWG above the guideline was associated with a lower risk of preterm birth ${ }^{(1)}$. However, in our study, the lowest quintile of GWG rate was associated with a higher risk of early preterm birth, while the highest quintile was associated with overall preterm, mainly due to its association with late preterm. Our findings were supported by a cohort study in Peru indicating a U- 
shaped association between GWG rate and the risk of preterm birth $^{(5)}$. One meta-analysis indicated that high total GWG was associated with a lower risk of preterm birth, while a high GWG rate was associated with a higher risk of preterm birth ${ }^{(24)}$. This discrepancy suggests that there may be some bias when using total GWG, because total GWG is inherently linked to gestational duration and, thus, to preterm birth. There are a number of possible biological mechanisms that may be involved in the association between GWG rate and preterm birth. A low GWG rate may be an indicator of micronutrient deficiency (such as vitamins $\mathrm{C}$ and $\mathrm{E}$ ) and reduced expansion of plasma volume, and the two latter conditions likely lead to preterm birth ${ }^{(25,26)}$. A high GWG rate is associated with altered placental microbiome profile, including decreased species richness and altered bacterially encoded metabolic pathways $^{(27)}$. A decreased species richness has been linked to insulin resistance, inflammatory phenotypes and infection and, thus, to preterm; altered bacterially encoded metabolic pathways, such as decreased abundance of folate biosynthesis and increased siderophore biosynthesis, were also linked to increased inflammation and worsened Fe status and, thus, to preterm $^{(28,29)}$.

Our study has some strengths. It was the first study to prospectively and comprehensively investigate the association of GWG rate in the second/third trimester with a range of pregnancy outcomes, including offspring mortality indicators, preterm birth and birth weight-related outcomes. Weekly GWG rate in the second/third trimester of pregnancy, rather than total GWG, was used as the exposure measure. GWG rate is likely more meaningful than total GWG in prenatal healthcare with respect to optimising weight gain during pregnancy. In addition, we adjusted for $\mathrm{Hb}$ and micronutrient status, which may be important confounders in the association between GWG and adverse pregnancy outcomes ${ }^{(30-32)}$.

However, our study also had a number of limitations. First, data on potential confounders, such as diet and physical activity ${ }^{(33,34)}$, were not collected, so we could not exclude the potential confounding effects. Furthermore, our sample size was relatively small, which limited our ability to perform detailed subgroup analyses according to BMI categories. Furthermore, using earlypregnancy BMI as the approximation of preconception BMI might lead to some women being misclassified, although such misclassification would probably be small. In addition, about $90 \%$ of women included in the study were farmers, which would likely restrict the generalisability of the results. Caution should be used when generalising the results to other ethnic populations, as our results were derived from a population mainly comprising Han Chinese women (99\%).

In conclusion, our study comprehensively investigated the association between GWG rate in second/third trimester and adverse pregnancy outcomes among Chinese nulliparous women. Very low and very high GWG rates were associated with increased risks of various adverse pregnancy outcomes, and very low GWG rates appeared to be particularly associated with mortality outcomes. Healthcare providers should integrate lifestyle and dietary interventions into routine healthcare during pregnancy to help women optimise their $\mathrm{GWG}^{(35,36)}$. Large-scale studies in Asian countries ${ }^{(37)}$ with more representative populations are needed to examine whether the associations observed here persist across BMI categories.

\section{Acknowledgements}

This work was supported by the National Key Research and Development Program of China (J. L., grant number 2016YFC1000401); the National Natural Science Foundation of China (J. L., grant number 81571517; Y. Z., grant number 81801542); and the Young Researcher Development Scheme of Peking University (Y. Z., grant number BMU20160571). The funders had no role in the conduct of this study.

J. L. had full access to all of the data in the study and takes responsibility for the integrity of the data and the accuracy of the data analysis. Study concept and design: Y. Z., J. L.; acquisition, analysis or interpretation of data: all authors; drafting of the manuscript: Y. Z.; critical revision of the manuscript for important intellectual content: all authors; statistical analysis: Y. Z.; administrative, technical or material support: all authors; study supervision: all authors.

There are no conflicts of interest.

\section{Supplementary material}

To view supplementary material for this article, please visit https://doi.org/10.1017/S0007114519001247

\section{References}

1. Goldstein RF, Abell SK, Ranasinha S, et al. (2017) Association of gestational weight gain with maternal and infant outcomes: a systematic review and meta-analysis. JAMA 317, 2207-2225.

2. Han Z, Lutsiv O, Mulla S, et al. (2011) Low gestational weight gain and the risk of preterm birth and low birthweight: a systematic review and meta-analyses. Acta Obstet Gynecol Scand 90, $935-954$.

3. Xu Z, Wen Z, Zhou Y, et al. (2017) Inadequate weight gain in obese women and the risk of small for gestational age (SGA): a systematic review and meta-analysis. J Matern Fetal Neonatal Med 30, 357-367.

4. Tian C, Hu C, He X, et al. (2016) Excessive weight gain during pregnancy and risk of macrosomia: a meta-analysis. Arch Gynecol Obstet 293, 29-35.

5. Carnero AM, Mejia CR \& Garcia PJ (2012) Rate of gestational weight gain, pre-pregnancy body mass index and preterm birth subtypes: a retrospective cohort study from Peru. BJOG 119, 924-935.

6. Chen A, Feresu SA, Fernandez C, et al. (2009) Maternal obesity and the risk of infant death in the United States. Epidemiology 20, 74-81.

7. Durie DE, Thornburg LL \& Glantz JC (2011) Effect of secondtrimester and third-trimester rate of gestational weight gain on maternal and neonatal outcomes. Obstet Gynecol 118, 569-575.

8. Huang A, Ji Z, Zhao W, et al. (2016) Rate of gestational weight gain and preterm birth in relation to prepregnancy body mass indices and trimester: a follow-up study in China. Reprod Health 13, 93.

9. Oken E, Kleinman KP, Belfort MB, et al. (2009) Associations of gestational weight gain with short- and longer-term maternal and child health outcomes. Am J Epidemiol 170, 173-180. 
10. Sharma AJ, Vesco KK, Bulkley J, et al. (2015) Associations of gestational weight gain with preterm birth among underweight and normal weight women. Matern Child Health $J \mathbf{1 9}$, 2066-2073.

11. Gilmore LA \& Redman LM (2015) Weight gain in pregnancy and application of the 2009 IOM guidelines: toward a uniform approach. Obesity 23, 507-511.

12. Bodnar LM, Siminerio LL, Himes KP, et al. (2016) Maternal obesity and gestational weight gain are risk factors for infant death. Obesity 24, 490-498.

13. Davis RR, Hofferth SL \& Shenassa ED (2014) Gestational weight gain and risk of infant death in the United States. Am J Public Health 104, Suppl. 1, S90-S95.

14. Friedmann I \& Balayla J (2018) Gestational weight gain and the risk of infant mortality amongst women with normal prepregnancy BMI: the Friedmann-Balayla model. J Matern Fetal Neonatal Med 31, 325-332.

15. Langford A, Joshu C, Chang JJ, et al. (2011) Does gestational weight gain affect the risk of adverse maternal and infant outcomes in overweight women? Matern Child Health J 15, 860-865.

16. Abrams B, Carmichael S \& Selvin S (1995) Factors associated with the pattern of maternal weight gain during pregnancy. Obstet Gynecol 86, 170-176.

17. Singh GK \& Yu SM (1996) Adverse pregnancy outcomes: differences between US- and foreign-born women in major US racial and ethnic groups. Am J Public Health 86, 837-843.

18. Liu JM, Mei Z, Ye R, et al. (2013) Micronutrient supplementation and pregnancy outcomes: double-blind randomized controlled trial in China. JAMA Intern Med 173, 276-282.

19. Institute of Medicine and National Research Council (2009) Weight Gain During Pregnancy: Reexamining the Guidelines. Washington, DC: National Academies Press. https://www. cbsnews.com/htdocs/pdf/052809_pregnancy.pdf (accessed September 2018).

20. WHO Expert Consultation (2004) Appropriate body-mass index for Asian populations and its implications for policy and intervention strategies. Lancet 363, 157-163.

21. Drehmer M, Duncan BB, Kac G, et al. (2013) Association of second and third trimester weight gain in pregnancy with maternal and fetal outcomes. PLOS ONE 8, e54704.

22. Oken E \& Gillman MW (2003) Fetal origins of obesity. Obes Res 11, 496-506.

23. Taylor PD \& Poston L (2007) Developmental programming of obesity in mammals. Exp Physiol 92, 287-298.
24. McDonald SD, Han Z, Mulla S, et al. (2011) High gestational weight gain and the risk of preterm birth and low birth weight: a systematic review and meta-analysis. J Obstet Gynaecol Can 33, 1223-1233.

25. Romero R, Chaiworapongsa $T$ \& Espinoza J (2003) Micronutrients and intrauterine infection, preterm birth and the fetal inflammatory response syndrome. J Nutr $\mathbf{1 3 3}$, 1668S-1673S.

26. Romero R, Dey SK \& Fisher SJ (2014) Preterm labor: one syndrome, many causes. Science 345, 760-765.

27. Antony KM, Ma J, Mitchell KB, et al. (2015) The preterm placental microbiome varies in association with excess maternal gestational weight gain. Am J Obstet Gynecol 212, 653.e1-e16.

28. Neilands JB (1995) Siderophores: structure and function of microbial iron transport compounds. J Biol Chem 270, 26723-26726.

29. Aagaard K, Ma J, Antony KM, et al. (2014) The placenta harbors a unique microbiome. Sci Transl Med 6, $237 \mathrm{ra} 65$.

30. Siega-Riz AM, Adair LS \& Hobel CJ (1996) Maternal underweight status and inadequate rate of weight gain during the third trimester of pregnancy increases the risk of preterm delivery. J Nutr 126, 146-153.

31. Scanlon KS, Yip R, Schieve LA, et al. (2000) High and low hemoglobin levels during pregnancy: differential risks for preterm birth and small for gestational age. Obstet Gynecol 96, 741-748.

32. Little MP, Brocard P, Elliott P, et al. (2005) Hemoglobin concentration in pregnancy and perinatal mortality: a London-based cohort study. Am J Obstet Gynecol 193, 220-226.

33. Stuebe AM, Oken E \& Gillman MW (2009) Associations of diet and physical activity during pregnancy with risk for excessive gestational weight gain. Am J Obstet Gynecol 201, 58.e1-e8.

34. Zhang C \& Ning Y (2011) Effect of dietary and lifestyle factors on the risk of gestational diabetes: review of epidemiologic evidence. Am J Clin Nutr 94, 1975s-1979s.

35. Guelinckx I, Devlieger R, Mullie P, et al. (2010) Effect of lifestyle intervention on dietary habits, physical activity, and gestational weight gain in obese pregnant women: a randomized controlled trial. Am J Clin Nutr 91, 373-380.

36. Thangaratinam S, Rogozinska E, Jolly K, et al. (2012) Effects of interventions in pregnancy on maternal weight and obstetric outcomes: meta-analysis of randomised evidence. BMJ $\mathbf{3 4 4}$, e2088.

37. Hedderson MM, Gunderson EP \& Ferrara A (2010) Gestational weight gain and risk of gestational diabetes mellitus. Obstet Gynecol 115, 597-604. 